\title{
Effect of Robotic-Assisted Gait Training in Patients With Incomplete Spinal Cord Injury
}

\author{
Ji Cheol Shin, MD, PhD, Ji Yong Kim, MD, Han Kyul Park, MD, Na Young Kim, MD \\ Department of Rehabilitation Medicine and Research Institute of Rehabilitation Medicine, \\ Yonsei University College of Medicine, Seoul, Korea
}

\begin{abstract}
Objective To determine the effect of robotic-assisted gait training (RAGT) compared to conventional overground training.

Methods Sixty patients with motor incomplete spinal cord injury (SCI) were included in a prospective, randomized clinical trial by comparing RAGT to conventional overground training. The RAGT group received RAGT three sessions per week at duration of 40 minutes with regular physiotherapy in 4 weeks. The conventional group underwent regular physiotherapy twice a day, 5 times a week. Main outcomes were lower extremity motor score of American Spinal Injury Association impairment scale (LEMS), ambulatory motor index (AMI), Spinal Cord Independence Measure III mobility section (SCIM3-M), and walking index for spinal cord injury version II (WISCI-II) scale.

Results At the end of rehabilitation, both groups showed significant improvement in LEMS, AMI, SCIM3-M, and WISCI-II. Based on WISCI-II, statistically significant improvement was observed in the RAGT group. For the remaining variables, no difference was found.

Conclusion RAGT combined with conventional physiotherapy could yield more improvement in ambulatory function than conventional therapy alone. RAGT should be considered as one additional tool to provide neuromuscular reeducation in patient with incomplete SCI.
\end{abstract}

Keywords Walking, Spinal cord injuries, Robotics, Recovery of function, Rehabilitation

Received July 1, 2014; Accepted August 11, 2014

Corresponding author: Na Young Kim

Department of Rehabilitation Medicine and Research Institute of Rehabilitation Medicine, Yonsei University College of Medicine, 50 Yonsei-ro, Seodaemun-gu, Seoul 120-749, Korea

Tel: +82-2-2227-2241, Fax: +82-2-364-0509, E-mail: kny8452@hanmail.net

(c) This is an open-access article distributed under the terms of the Creative Commons Attribution Non-Commercial License (http://creativecommons. org/licenses/by-nc/3.0) which permits unrestricted noncommercial use, distribution, and reproduction in any medium, provided the original work is properly cited.

Copyright () 2014 by Korean Academy of Rehabilitation Medicine

\section{INTRODUCTION}

Recovery of walking ability is one of the main goals of patients after spinal cord injury (SCI), a factor associated with quality of life and satisfaction [1]. Recently, more than $50 \%$ of people with SCI have motor incomplete lesions. The proportion of incomplete SCI has been increasing [2]. Most motor recovery occurs within two months after injury. More than $75 \%$ of patients with initial motor incomplete SCI regain some form of ambulatory function. American Spinal Injury Association (ASIA) impairment scale grade D patients have a very good 
prognosis at 1 -year post-injury $[3,4]$.

However, successful gait training for SCI patients is costly. In addition, it requires space and skilled physiotherapists who can consider diverse aspects of physical status of the patient. The main limitations of overground walking ability for patients with SCI are reduced sensorymotor coordination, spasticity, impaired balance [5], as well as muscle weakness. Practically, there are numerous constraints in the provision of an individualized training strategy. Various treatments have been attempted to facilitate walking ability, such as robotic-assisted gait training (RAGT), for SCI patients.

Recently implemented treatments have been focused on enhancing the activity of spinal interneurons based on function of the central pattern generator by providing sensory-motor stimulation to optimize neural plasticity [6]. RAGT is also based on this strategy. It has the advantage of repeatedly conducting a pre-programmed gait pattern. Several studies provide evidence that RAGT promotes motor recovery and functional improvement $[7,8]$. However, other studies demonstrated that there was no significant difference between RAGT and conventional therapy [9]. Several systematic reviews including a Cochrane review article [10-12] found that there was insufficient evidence to determine the superiority of one gait training strategy over another. Further investigation is needed to determine strategies that could result in the best response and optimal training.

\section{MATERIALS AND METHODS}

\section{Participants}

Between May 2012 and May 2014, all SCI patients admitted to the Department of Physical Medicine and Rehabilitation of Severance Hospital were evaluated and selected when they met the following inclusion criteria: 1) non-progressive spinal cord lesion as a result of traumatic or non-traumatic causes, 2) onset less than 6 months, 3) classified by the ASIA impairment scale (AIS) as grade D at entry, and 4) 20 to 65 years old. Exclusion criteria were patients with pressure ulcers, severe limitation of range of motion of the hips and knee joints, severe cognitive impairment, or patients with pulmonary or heart disease requiring monitoring during exercise. Patients were also excluded if they had lower motor neuron lesion, such as cauda equina injury, or had previously experi- enced RAGT. A total of 60 participants were enrolled and randomly assigned to the RAGT group or conventional group. Baseline measurements of outcome variables were taken before they underwent intervention.

\section{Robotic-assisted gait training system}

The Lokomat system (Hocoma AG, Zurich, Switzerland) included a treadmill, a body-weight support system, and two lightweight robotic actuators attached to subject's legs (Fig. 1). The speed of the treadmill could be adjusted from $0 \mathrm{~km} / \mathrm{hr}$ to approximately $3 \mathrm{~km} / \mathrm{hr}$. During treatments, velocity of the treadmill was fixed at $1.5 \mathrm{~km} / \mathrm{hr}$. At the beginning of the treatment, approximately $50 \%$ of each subject's body weight was supported by the harness system. During the following walking sessions, the bodyweight support was reduced to the minimum as tolerated without substantial knee buckling or toe drag. Guidance force was maintained at $100 \%$.

\section{Intervention}

All patients assigned to each treatment group were treated with physical therapy in 4 weeks. The RAGT group received RAGT with regular physiotherapy in the following schedule: 3 days with RAGT and 2 days with regular treatment a week. On the day receiving RAGT, patients performed one RAGT session with one regular physiotherapy session. The overall session time of RAGT

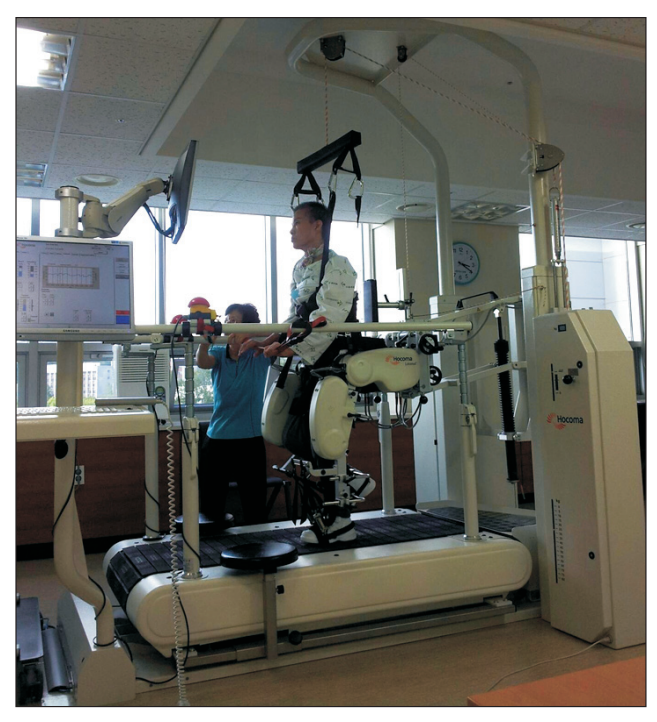

Fig. 1. Lokomat, a robot-assisted gait training system, consisted of robotic gait orthosis, body weight support, and treadmill. 
treatment was 1-hour, including set-up time. The actual training time was 40 minutes. In the rest treatment days, subjects underwent regular physiotherapy twice a day in a 30-minute session. The control group underwent regular physiotherapy twice a day and 5 times a week using Bobath principles. All subjects were allowed to participate in other treatments, such as occupational therapy or functional electrical stimulation (FES), during the duration of the study.

\section{Outcome measures}

All participants were assessed within 48 hours of starting and the end of training period. The muscular strength of the lower limb was measured with ASIA lower extremity motor score subscale (LEMS; range 0 to 50 ) and the ambulatory motor index (AMI; range 0 to 30 ). The ability to walk was evaluated according to the outdoor and indoor mobility domain of the Spinal Cord Independence Measurement III mobility section (SCIM3-M; range 0 to 30 ) and the walking index for spinal cord injury version II (WISCI-II). WISCI-II was a 20 -item scale measuring the walking status of a patient based on the requirements of assistance and/or bracing and/or walking aids.

\section{Statistical analysis}

The general characteristics or baseline data were compared between each group by using Student t-test (for continuous variables) if data were normally distributed. A Wilcoxon test was used for non-normally distributed ordinal scale. The associations between qualitative variables were assessed by $\chi^{2}$-test. Within-group comparisons of treatment effects relative to baseline were carried out with Wilcoxon signed-rank test. Betweengroup comparisons were performed with Mann-Whitney U test. SPSS ver. 21.0 software (IBM SPSS Inc., Armonk, NY, USA) was used for the statistical analyses. Statistical significance was considered when $\mathrm{p}$-value was less than 0.05 .

\section{RESULTS}

\section{Demographic data}

A total of 456 patients were assessed for eligibility, of which 60 who met the inclusion criteria were enrolled in this study. Seven withdrawals occurred, including three in the RAGT group and four in the conventional group.
The reasons for withdrawals were not associated with the study. Finally, a total of 53 participants were analyzed (Fig. 2). Demographic and injury data for each group along with baseline results are summarized in Table 1 . No significant difference was detected in age, sex, injury level, time course, or treatment periods. Therefore, the study groups were comparable.

Muscular strength and gait abilities improvement during study

There was no significant difference in baseline measurement. At the end of the rehabilitation period, each group showed significant $(\mathrm{p}<0.05)$ recovery in muscular strength according to the LEMS and the AMI scale. The RAGT group showed more improvement in the AMI with borderline significance $(\mathrm{p}=0.06)$. The AMI improved from 21 (interquartile range [IQR], 10-30) to 25 (IQR, 15-30)

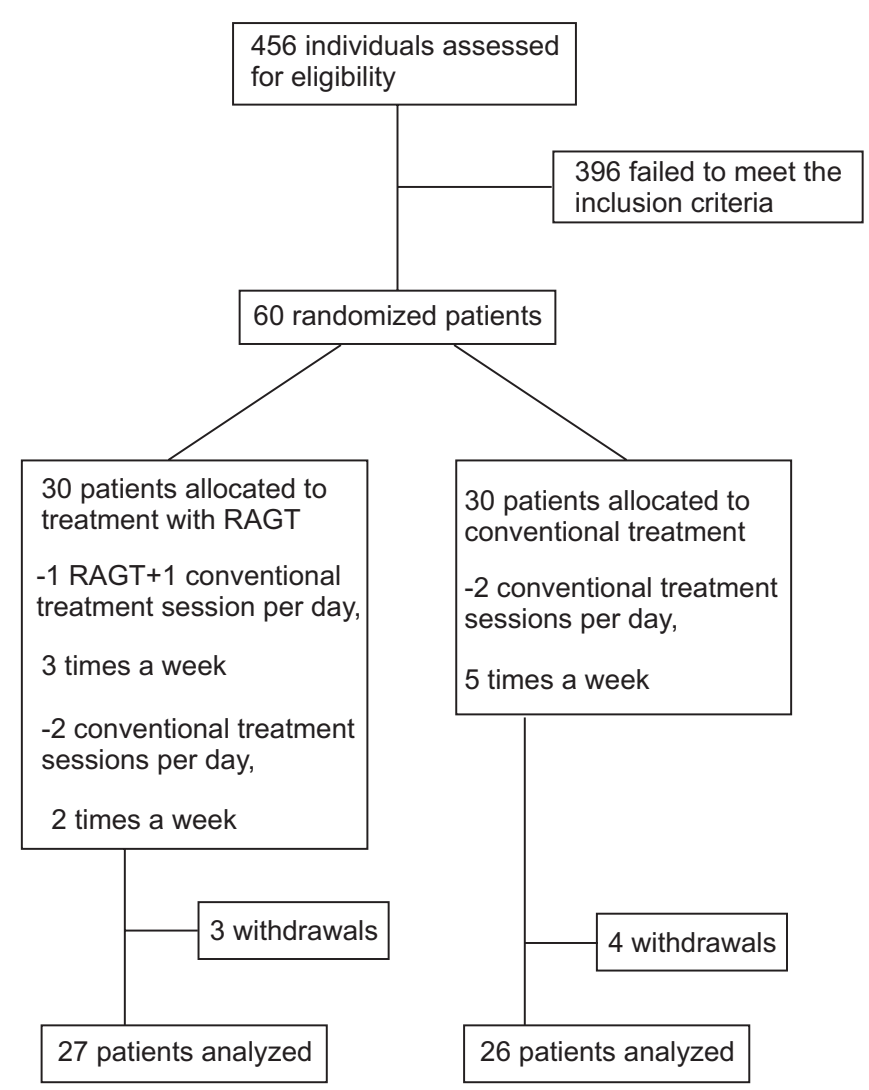

Fig. 2. A total of 456 patients with spinal cord injury were assessed from May 2012 to May 2014. A total of 60 patients who had non-progressive spinal cord lesion elapsed less than 6 months were randomly allocated. RAGT, roboticassisted gait training. 
Table 1. Participant baseline characteristics

\begin{tabular}{|c|c|c|c|}
\hline & Conventional $(\mathrm{n}=\mathbf{2 6})$ & RAGT + conventional $(n=27)$ & p-value \\
\hline Age (yr) & $48.15 \pm 11.49$ & $43.15 \pm 14.37$ & 0.17 \\
\hline Sex & & & 0.16 \\
\hline Male & $14(53.8)$ & $20(74.1)$ & \\
\hline Female & $12(46.2)$ & $7(25.9)$ & \\
\hline Injury level & & & 0.48 \\
\hline Cervical & $16(61.5)$ & $15(51.9)$ & \\
\hline Thoracic \& lumbar & $10(38.5)$ & $12(48.1)$ & \\
\hline Etiology & & & 0.77 \\
\hline Traumatic & $19(73.1)$ & $18(66.7)$ & \\
\hline Non-traumatic & $7(26.9)$ & $9(33.3)$ & \\
\hline Time from onset (month) & $2.73 \pm 1.97$ & $3.33 \pm 2.02$ & 0.17 \\
\hline Treatment period (day) & $30.73 \pm 3.07$ & $30.42 \pm 3.70$ & 0.53 \\
\hline
\end{tabular}

Values are presented as mean \pm standard deviation or number (\%).

RAGT, robotic-assisted gait training.

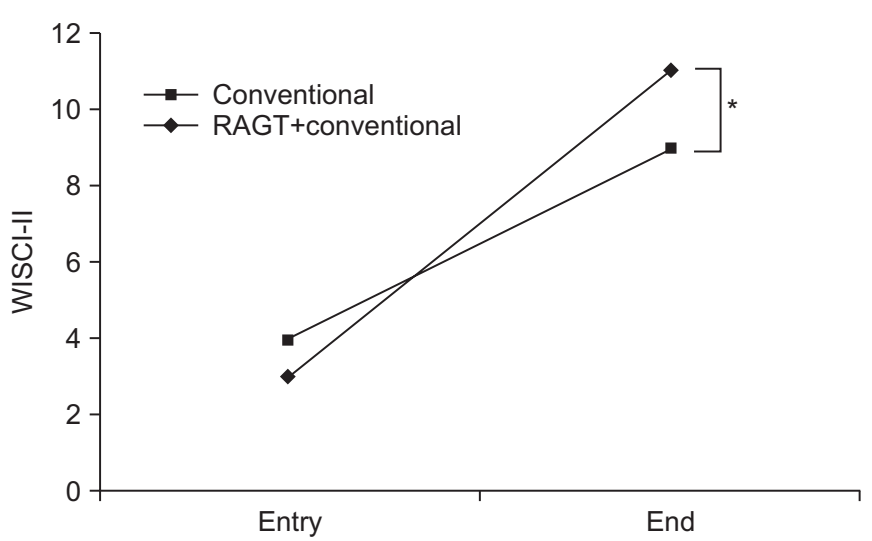

Fig. 3. Line graphs showing scores of gait abilities evaluated by WISCI-II at entry and the end of the treatment in the RAGT+conventional or conventional groups. RAGT patients showed significant greater gain in WISCI-II compared to those in the conventional group. RAGT, roboticassisted gait training; WISCI-II, walking index for spinal cord injury version II. * $\mathrm{p}<0.05$.

in the RAGT group, which was improved from 23 (IQR, 13-30) to 26 (IQR, 16-30) in the conventional group.

Each group achieved a significant improvement in ambulation ability based on SCIM3-M and WISCI-II (Table 2, Fig. 3). Patients in the RAGT group showed significant greater gain (from 3 [IQR, 0-14] to 11 [IQR, 0-19]) compared to controls in the WISCI-II (from 4 [IQR, 0-16] to 9 [IQR, 0-20]). The variances of outcome measures were summarized in Table 2.

\section{DISCUSSION}

In this study, a SCI patient graded AIS-D showed significant improvement in muscle strength and functional recovery after an average of 4 weeks of treatment, with favorable outcome showing in the RAGT group. The requirements of assistance were significantly reduced based on WISCI-II which was in favor of walking reeducation with RAGT. RAGT was not performed to completely replace conventional therapy. RAGT provided objective, repetitive, and qualitatively equal treatment. However, it could not reflect an immediate feedback. Therefore, RAGT combined with conventional physiotherapy could produce additive benefit over conventional physiotherapy alone.

In RCT study involving subacute SCI patients within 3 to 6 months of incomplete spinal cord lesion, Alcobendas-Maestro et al. [7] found that RAGT groups improved significantly from baseline to follow-up with respect to FIM-L, WISCI-II, LEMS, and walk distance. Our study has many similarities to the study of Alcobendas-Maestro et al. [7], including subject population and treatment protocol. However, we did not find difference between groups regarding the achievement of recovery based on LEMS and AMI scores. Prior to concluding that RAGT had no effect on restoring muscle strength or functional level, three possibilities should be considered. Firstly, RAGT was performed with fixed guidance force and walking velocity in our study. Therefore, neural plasticity might 
Table 2. Muscular strength and gait abilities at entry and the end of the treatment

\begin{tabular}{|c|c|c|c|c|c|c|c|c|c|}
\hline & \multicolumn{4}{|c|}{ Conventional (n=26) } & \multicolumn{4}{|c|}{ RAGT + conventional $(n=27)$} & \multirow{2}{*}{ p-value ${ }^{b}$} \\
\hline & Entry & End & Variance & p-value ${ }^{a)}$ & Entry & End & Variance & p-value ${ }^{\text {a) }}$ & \\
\hline LEMS & $33(20-40)$ & $37(20-48)$ & $4(0-18)$ & $<0.001^{* * *}$ & $31(12-40)$ & $37(20-49)$ & $6(0-18)$ & $<0.001^{* * *}$ & 0.24 \\
\hline AMI & $23(13-30)$ & $26(16-30)$ & $2(0-10)$ & $<0.001^{* * *}$ & $21(10-30)$ & $25(15-30)$ & $4(0-22)$ & $<0.001^{* * *}$ & 0.06 \\
\hline SCIM3-M & $6(0-18)$ & $9(0-33)$ & $3(0-24)$ & $<0.001^{* * *}$ & $4(0-11)$ & $10(0-26)$ & $6(0-20)$ & $<0.001^{* * *}$ & 0.13 \\
\hline WISCI-II & $4(0-16)$ & $9(0-20)$ & $5(0-20)$ & $<0.001^{* * *}$ & $3(0-14)$ & $11(0-19)$ & $8(0-17)$ & $<0.001^{* * *}$ & $0.01^{*}$ \\
\hline
\end{tabular}

Values are presented as median (interquartile range).

LEMS, lower extremity motor score; AMI, ambulatory motor index; SCIM3-M, mobility domain of the Spinal Cord Independence Measurement-III; WISCI-II, walking index for spinal cord injury.

${ }^{\text {a) }}$ For intragroup comparison, ${ }^{\text {b) }}$ for intergroup comparison. ${ }^{*} \mathrm{p}<0.05,{ }^{* * *} \mathrm{p}<0.001$.

have been impeded by the limitation of voluntary movement. Movement variability is thought to be a critical feature underlying motor learning [13]. A comparable study is needed. Secondly, the assessment tools may be inappropriate. Walking ability should be measured based on both spatial and temporal parameters as well as kinematics (joint range changes) and kinetics (moments and forces acting across joints). RAGT can significantly reduce neuromuscular abnormalities associated with spasticity [14]. RAGT can also promote intralimb and interlimb coordination and alter co-contraction between knee and ankle antagonistic muscles [15]. Lastly, difference in treatment duration could yield different outcomes.

A recent study using a very intensive (3 hours per day, 5 times in a week for 2 weeks) training schedule for individuals did not result in changes in walking speed over ground [16]. Considering that RAGT was performed at least 8 weeks in other studies and that those studies concluded that RAGT was more effective than conventional therapy $[7,8]$, continuation may be more important than intensity or frequency of treatment to gain better outcome.

In this study, 17 of 27 patients could not stand alone who needed more than two assistants to perform gait training. Fourteen of 27 patients started RAGT less than 3 months after the onset of SCI, whereas previous studies examined subacute $[7,8]$ or chronic SCI patients [1721] who were able to use RAGT on their own without any safety issues. Participants who started earlier after injury ( $<4$ weeks) showed significantly faster walking speeds and walking distance than those started later [22]. Patients who started training $<6$ months post-injury ended up with higher final walking scores than those who started $>6$ months post-injury [23]. Therefore, earlier walking training could be more effective. Furthermore, participating in gait training without support has a significant potential to improve self-image and positive change of emotion [24,25], regardless of the physical improvement [26]. RAGT also improves confidence in walking performance [21]. Considering the psychological benefits and its effect from early intervention, RAGT might be considered for implementation before overground walking training.

Our study had several limitations. The number of patients per group was relatively small with diverse etiologies. Only short-term outcome was evaluated. Longterm follow-up was not assessed. Our results showed that RAGT could be an effective supplement for gait training in SCI patients. However, it did not afford a basis on which we could claim that this kind of training was better than traditional technique. Nonetheless, this study adds to the wealth of data necessary to determine appropriate candidate, optimal timing, and the best protocol to design maximal efficacy of RAGT in SCI patients.

In conclusion, RAGT combined with conventional physiotherapy could yield more improvement in ambulatory function than conventional treatment alone, therefore improving muscle strength and walking ability in motor incomplete SCI patients. RAGT should be considered as one of the training methods to provide safe and effective way to perform neuromuscular re-education for SCI patients.

\section{CONFLICT OF INTEREST}

No potential conflict of interest relevant to this article was reported. 


\section{REFERENCES}

1. van Middendorp JJ, Hosman AJ, Van de Meent H. Who wants to walk? Preferences for recovery after SCI: a longitudinal and cross-sectional study. Spinal Cord 2009;47:268-9.

2. Shin JC, Kim DH, Yu SJ, Yang HE, Yoon SY. Epidemiologic change of patients with spinal cord injury. Ann Rehabil Med 2013;37:50-6.

3. Burns SP, Golding DG, Rolle WA Jr, Graziani V, Ditunno JF Jr. Recovery of ambulation in motor-incomplete tetraplegia. Arch Phys Med Rehabil 1997;78:1169-72.

4. van Middendorp JJ, Hosman AJ, Pouw MH; EM-SCI Study Group, Van de Meent H. ASIA impairment scale conversion in traumatic SCI: is it related with the ability to walk? A descriptive comparison with functional ambulation outcome measures in 273 patients. Spinal Cord 2009;47:555-60.

5. Dietz V, Colombo G, Jensen L, Baumgartner L. Locomotor capacity of spinal cord in paraplegic patients. Ann Neurol 1995;37:574-82.

6. AuYong N, Lu DC. Neuromodulation of the lumbar spinal locomotor circuit. Neurosurg Clin N Am 2014;25:15-23..

7. Alcobendas-Maestro M, Esclarin-Ruz A, CasadoLopez RM, Munoz-Gonzalez A, Perez-Mateos G, Gonzalez-Valdizan E, et al. Lokomat robotic-assisted versus overground training within 3 to 6 months of incomplete spinal cord lesion: randomized controlled trial. Neurorehabil Neural Repair 2012;26:1058-63.

8. Schwartz I, Sajina A, Neeb M, Fisher I, Katz-Luerer M, Meiner Z. Locomotor training using a robotic device in patients with subacute spinal cord injury. Spinal Cord 2011;49:1062-7.

9. Field-Fote EC, Roach KE. Influence of a locomotor training approach on walking speed and distance in people with chronic spinal cord injury: a randomized clinical trial. Phys Ther 2011;91:48-60.

10. Mehrholz J, Kugler J, Pohl M. Locomotor training for walking after spinal cord injury. Cochrane Database Syst Rev 2012;11:CD006676.

11. Swinnen E, Duerinck S, Baeyens JP, Meeusen R, Kerckhofs E. Effectiveness of robot-assisted gait training in persons with spinal cord injury: a systematic review. J Rehabil Med 2010;42:520-6.

12. Morawietz C, Moffat F. Effects of locomotor training after incomplete spinal cord injury: a systematic review. Arch Phys Med Rehabil 2013;94:2297-308.

13. Lennon S, Baxter D, Ashburn A. Physiotherapy based on the Bobath concept in stroke rehabilitation: a survey within the UK. Disabil Rehabil 2001;23:254-62.

14. Mirbagheri MM, Kindig M, Niu X, Varoqui D, Conaway P. Robotic-locomotor training as a tool to reduce neuromuscular abnormality in spinal cord injury: the application of system identification and advanced longitudinal modeling. IEEE Int Conf Rehabil Robot 2013;2013:6650497.

15. Knikou M, Mummidisetty CK. Locomotor training improves premotoneuronal control after chronic spinal cord injury. J Neurophysiol 2014;111:2264-75.

16. Fritz SL, Merlo-Rains AM, Rivers ED, Peters DM, Goodman A, Watson ET, et al. An intensive intervention for improving gait, balance, and mobility in individuals with chronic incomplete spinal cord injury: a pilot study of activity tolerance and benefits. Arch Phys Med Rehabil 2011;92:1776-84.

17. Varoqui D, Niu X, Mirbagheri MM. Ankle voluntary movement enhancement following robotic-assisted locomotor training in spinal cord injury. J Neuroeng Rehabil 2014;11:46.

18. Wirz M, Zemon DH, Rupp R, Scheel A, Colombo G, Dietz V, et al. Effectiveness of automated locomotor training in patients with chronic incomplete spinal cord injury: a multicenter trial. Arch Phys Med Rehabil 2005;86:672-80.

19. Mirbagheri MM, Niu X, Kindig M, Varoqui D. The effects of locomotor training with a robotic-gait orthosis (Lokomat) on neuromuscular properties in persons with chronic SCI. Conf Proc IEEE Eng Med Biol Soc 2012;2012:3854-7.

20. Labruyere R, van Hedel HJ. Strength training versus robot-assisted gait training after incomplete spinal cord injury: a randomized pilot study in patients depending on walking assistance. J Neuroeng Rehabil 2014;11:4.

21. Lam T, Pauhl K, Krassioukov A, Eng JJ. Using robotapplied resistance to augment body-weight-supported treadmill training in an individual with incomplete spinal cord injury. Phys Ther 2011;91:143-51.

22. Dobkin B, Apple D, Barbeau H, Basso M, Behrman A, Deforge D, et al. Weight-supported treadmill vs overground training for walking after acute incomplete 
SCI. Neurology 2006;66:484-93.

23. Benito-Penalva J, Edwards DJ, Opisso E, Cortes M, Lopez-Blazquez R, Murillo N, et al. Gait training in human spinal cord injury using electromechanical systems: effect of device type and patient characteristics. Arch Phys Med Rehabil 2012;93:404-12.

24. Semerjian TZ, Montague SM, Dominguez JF, Davidian AM, de Leon RD. Enhancement of quality of life and body satisfaction through the use of adapted exercise devices for individuals with spinal cord injuries. Top Spinal Cord Inj Rehabil 2005;11:95-108.
25. Martin Ginis KA, Latimer AE. The effects of single bouts of body-weight supported treadmill training on the feeling states of people with spinal cord injury. Spinal Cord 2007;45:112-5.

26. Hicks AL, Adams MM, Martin Ginis K, Giangregorio L, Latimer A, Phillips SM, et al. Long-term body-weightsupported treadmill training and subsequent followup in persons with chronic SCI: effects on functional walking ability and measures of subjective well-being. Spinal Cord 2005;43:291-8. 